\title{
Numerical Simulation of Skin Formation: The Relationship between Transepidermal Water Loss and Corneum Thickness
}

\author{
Katsuya Nagayama, Takeshi Kurihara \\ Department of Mechanical Information Science and Technology, Kyushu Institute of Technology, Fukuoka, Japan \\ Email: nagayama@mse.kyutech.ac.jp
}

How to cite this paper: Nagayama, K. and Kurihara, T. (2018) Numerical Simulation of Skin Formation: The Relationship between Transepidermal Water Loss and Corneum Thickness. Journal of Applied Mathematics and Physics, 6, 1757-1762. https://doi.org/10.4236/jamp.2018.68150

Received: April 24, 2018

Accepted: August 28, 2018

Published: August 31, 2018

\begin{abstract}
Skin moisturizing has drawn attention in terms of beauty and anti-aging industries. However, it is difficult to observe the inside of the epidermis and the relationship between the epidermis and water content is not yet clear. Computational simulations can be useful in understanding such mechanisms of skin formation. A particle model was used to simulate three-dimensional skin turnover, and the results reproduced the epidermal skin turnover phenomenon. In this study, a diffusion model is introduced into this simulation model and a moisture diffusion analysis of the epidermis was performed. In particular, transepidermal water loss (TEWL) was modeled by considering diffusion and surface evaporation in the stratum corneum and other layers. The relationship between the moisture content and the keratin detachment was considered, and the exfoliation condition of keratin based on the moisture content was calculated in the model. As a result, it was possible to calculate the intraepidermal water content distribution in the skin using the particle model. It was also possible to reproduce phenomena such as keratin thickening due to increase of TEWL. This phenomenon is consistent with cases of dry skin. In the future, it will be necessary to introduce a change in TEWL according to the thickness of the stratum corneum and the diffusion coefficient.
\end{abstract}

\section{Keywords}

Numerical Simulation, Particle Model, Stratum Corneum, TEWL, Diffusion Equation, Moisture Retention

\section{Introduction}

Skin moisturizing is closely related to the moisture content of the entire epider- 
mis, and it affects the appearance of the skin, e.g., facial wrinkles and firmness. In particular, because the stratum corneum is located on the outermost side of the skin, it is possible to control moisture evaporation on the skin surface and maintain normal skin via proper moisturizing care. In addition, in recent years, much attention has been given to the anti-aging and beauty industries and anti-aging effects due to continuous moisturizing care are expected.

Keratin intercellular connections are related to moisture content; accordingly, the peeling of keratin and its thickness likely depend on the amount of moisture. The epidermis containing keratin is composed of four layers with different properties, the bottom of the bottom layer and the dermis under the epidermis supply moisture to the upper part of the epidermis. However, it is difficult to observe the inside of the epidermis and the relationship between the epidermis and the water content is not yet clear.

Computational simulations can be useful in understanding the mechanisms underpinning the development of human skin, and several models have been proposed [1] [2] [3] [4]. We proposed a particle model that can handle complex biological phenomena, including cell interactions such as cell division, motion, deformation, and transition [5] [6]. This model was applied to the formation and turnover process of skin [7]. The model was further applied to basal layer formation and could reproduce young skin and aging skin [8] [9].

In this study, a moisture diffusion model is added to this simulation model [7] [8] [9]. TEWL (transepidermal water loss) was considered in the diffusion coefficient, and surface evaporation was considered in the stratum corneum and other layers. The model can simulate the intraepidermal water content distribution and the structure in the skin.

\section{Analysis Object and Model Description}

\subsection{Analysis Object}

Figure 1 depicts a cross section of the skin [10], and the roles of each cell layer are described here. The epidermis is the outermost layer of the skin and is primarily composed of cells called keratinocytes. The epidermis consists of four layers. The lowest layer of the epidermis, the basal layer, provides new cells each day by dividing. The dividing cells are called the prickle cell layer, and they are pushed and moved up toward the skin surface. This layer transforms into the

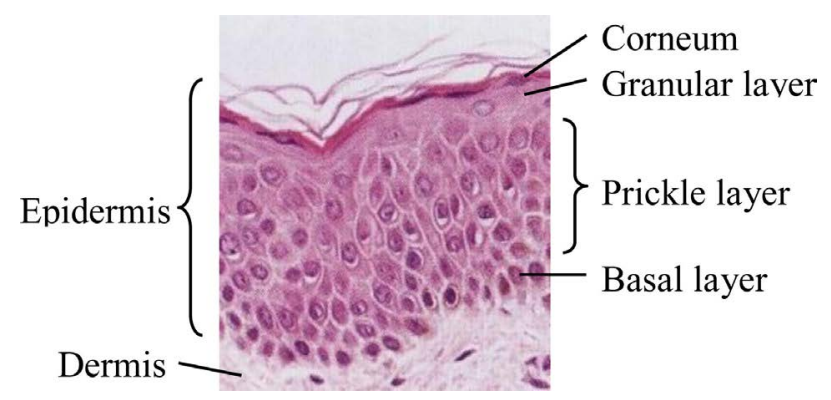

Figure 1. Cross-section of the skin [10]. 
granular layer and then the stratum corneum, which detaches itself from the surface of the skin. This is called the turnover process and occurs approximately every four weeks. During this turnover process, skin cells change not only in their shape but also in their physical properties. Here intercellular adhesion substances such as desmosomes are affected by the exfoliation of keratin and it is likely that water content is related to this decomposition.

The dermis is located under the epidermis, and the two are separated by the basal layer. Capillaries in the dermis supply nutrition and oxygen to the basal cells. Therefore, it is thought that the epidermis is influenced by force interactions from the dermis and nutrition effects from capillaries.

\subsection{Model Description}

The particle model [5] [6] was introduced to simulate the epidermis formation process [7] [8] [9]. The model considers the interactions between the particles and follows the motions of the particles in a Lagrangian manner. This method is suitable for analyses with large deformations, or when the numbers of the calculation points are changing. The cellular particles move in response to inter-particle forces, such as the force of conservation of volume and the spring force. The force of conservation of volume works to maintain the distances between the particles. Due to the repulsive force, particles eventually move to a stable distance. The spring force works to make the continuum of the cellular particles structural at the stratum corneum and the granular layer [7] [8] [9]. By summing up the forces from the surrounding particles, the particles gradually move to a position of force balance.

By introducing a diffusion model, it is possible to analyze the water content of the epidermis. The diffusion equation to be used is shown in Equation (1). In this analysis, because moisture in the epidermis is assumed to be steady state, the time terms are not considered. Here, $\mathrm{x}$ is the vertical direction in the epidermis, $\mathrm{y}$ and $\mathrm{z}$ are the plane directions in the epidermis, $\mathrm{D}$ represents the diffusion coefficient, and $\mathrm{C}$ represents the water concentration.

$$
D\left(\frac{\partial^{2} C}{\partial x^{2}}+\frac{\partial^{2} C}{\partial y^{2}}+\frac{\partial^{2} C}{\partial z^{2}}\right)=0
$$

In this case, the change in the moisture is only considered in the vertical direction and is assumed to be uniform in the surface direction. Here, the moisture evaporation amount of the skin is usually evaluated by TEWL. TEWL $\left[\mathrm{g} /\left(\mathrm{m}^{2} \mathrm{~h}\right)\right]$ refers to the weight $[\mathrm{g}]$ of moisture per unit time $[\mathrm{h}]$ per unit area $\left[\mathrm{m}^{2}\right]$, that is, the amount of moisture that evaporates from $1 \mathrm{~m}^{2}$ of skin surface per hour. TEWL and the integral of Equation (1) have the following relationship.

$$
T E W L=-D \frac{d C}{d x}
$$

The diffusion coefficient of moisture differs between corneum and other layers. Therefore, assuming that the diffusion coefficients are $D_{1}$ (corneum) and $D_{2}$ 
(other layers) and the moisture amounts are $C_{1}$ and $C_{2}$, and their relations with TEWL is expressed as the following Equations ((3) and (4)), respectively:

$$
\begin{aligned}
\frac{d C_{1}}{d x} & =-\frac{T E W L}{D_{1}} \\
\frac{d C_{2}}{d x} & =-\frac{T E W L}{D_{2}}
\end{aligned}
$$

\section{Calculation Conditions}

Cell particles in this model are represented by spherical shapes with diameters of $10 \mu \mathrm{m}$. The shape becomes thinner in the granular layer, changing with time, and adopts an elliptical shape that extends in the transverse direction by approximately $1 \mu \mathrm{m}$ in thickness in the stratum corneum.

The basal layer has 900 cells (in a $30 \times 30$ particle square) initially. Each basal cell divides once every five days of analysis time ( 0.2 times per day). The divided prickle cells freely move under the force of conservation of volume until they reach the granular layer. The spring force begins to gradually occur from the granular layer to the stratum corneum and connects to each particle. Consequently, migrations are reduced and the structure becomes fixed.

The diffusion coefficients at corneum $\left(D_{1}\right)$ and those at other layers $\left(D_{2}\right)$ are set $1.0 \times 10^{-9}\left[\mathrm{~cm}^{2} / \mathrm{s}\right]$ and $3.5 \times 10^{-5}\left[\mathrm{~cm}^{2} / \mathrm{s}\right]$, respectively; these are typical values for healthy skin [11] [12]. In addition, $60 \%-80 \%$ of the weight of the dermis is moisture. Therefore, 0.6 times the water density $1.0\left[\mathrm{~g} / \mathrm{cm}^{3}\right]$ was taken as the water concentration of the dermis and basal layer (the boundary condition). Under these conditions, the moisture content was calculated every day using Equations (3) and (4).

Further, there is nearly a complete correlation between the moisture content of keratinocytes and the presence rate of keratin adhesive (desmosome) [13]. Therefore, the existence ratio of the horny adhesive substance was determined from the moisture content of the keratinocyte and, when the value was 68.77 [\%] or less, the horny layer peeled off in 7 days. In addition, when the presence rate of the horny adhesive substance was larger than 68.77 [\%], depending on the value, it was set so as to exfoliate the horny layer over a maximum of 14 days [14]. Under the above conditions, the evaporation amount was increased to 3.0, 4.0 , and $5.0\left[\mathrm{~g} /\left(\mathrm{m}^{2} \mathrm{~h}\right)\right]$ and the thickness of the stratum corneum and the water content at the top of the horny layer were analyzed.

\section{Results}

Figure 2 shows each layer and the water content coded by color.

As TEWL increases, the horny material becomes thicker and the moisture content on the keratinous surface decreases. These trends are particularly noticeable when TEWL is $5\left[\mathrm{~g} /\left(\mathrm{m}^{2} \mathrm{~h}\right)\right]$.

Table 1 shows the changes in thickness and moisture content of the stratum corneum in the keratolytic model. The larger the amount of evaporation, the 


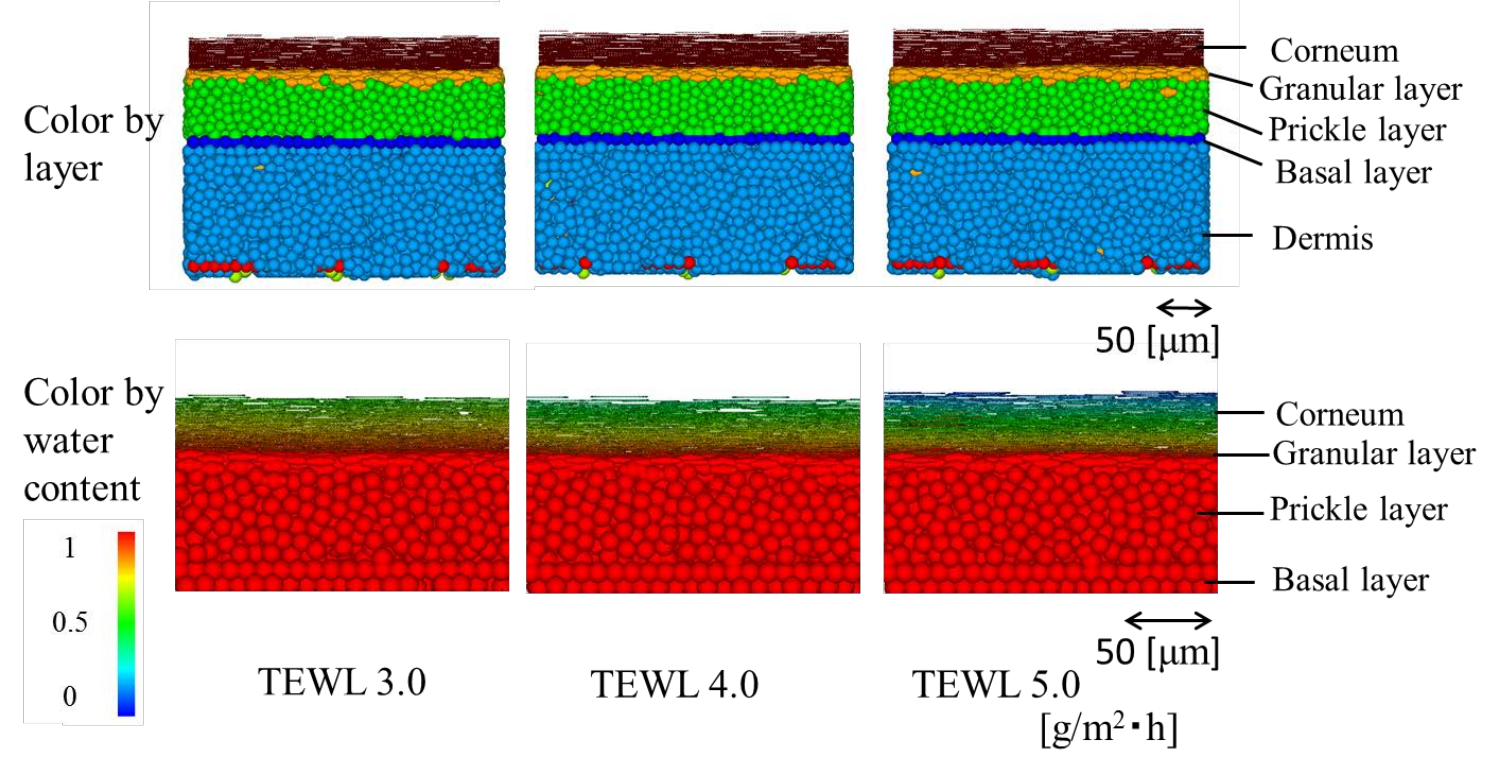

Figure 2. Surface cross-section results when changing TEWL (color-coded by layer and moisture content).

Table 1. Thickness of the stratum corneum and the moisture content.

\begin{tabular}{cccc}
\hline TEWL $\left[\mathrm{g} /\left(\mathrm{m}^{2} \mathrm{~h}\right]\right.$ & 3 & 4 & 5 \\
Corneum thickness $[\mu \mathrm{m}]$ & 30.7 & 30.9 & 33.3 \\
Water content $[\%]$ & 57.8 & 43.7 & 10 \\
\hline
\end{tabular}

greater the loss of water from the epidermis, which indicates dry skin. In this analysis result, it can be confirmed that as the skin dries, the stratum corneum becomes thicker. This is because the moisture content of the stratum corneum has decreased and the presence rate of the keratinous adhesive substance has increased. That is, the keratinocytes are difficult to peel off and the number of days until peeling increases. As an actual phenomenon, evaporation of moisture from the epidermis is prevented when the stratum corneum layer becomes thick and the evaporation amount decreases. In the future, it will be necessary to introduce a change in the amount of evaporation according to the thickness of the stratum corneum and the diffusion coefficient.

\section{Conclusion}

In this study, numerical simulations of epidermis formation using a particle model were performed. Adding the models of water diffusion and Corneum exfoliation, it was possible to calculate the water content in the epidermis. As a result of introducing the evaporation amount (TEWL) and the diffusion coefficient as a parameter to decide the water content, it was possible to reproduce phenomena such as the thickening of keratin in a dry state due to increase of TEWL. This phenomenon is consistent with cases of dry skin. In the future, it will be necessary to introduce a change in TEWL according to the thickness of the stratum corneum and the diffusion coefficient. 


\section{Conflicts of Interest}

The authors declare no conflicts of interest regarding the publication of this paper.

\section{References}

[1] Querleux, B. (2014) Computational Biophysics of the Skin. Pan Stanford Publishing. https://doi.org/10.1201/b17205

[2] Sütterlin, T., Huber, S., Dickhaus, H. and Grabe, N. (2009) Modeling Multi-Cellular Behavior in Epidermal Tissue Homeostasis via Finite State Machines in Multi-Agent Systems. Bioinformatics, 25, 2057-2063. https://doi.org/10.1093/bioinformatics/btp361

[3] Hirashima, T., Hosokawa, Y., Iino, T. and Nagayama, M. (2013) On Fundamental Cellular Processes for Emergence of Collective Epithelial Movement. Biology Open, 2, 600-666. https://doi.org/10.1242/bio.20134523

[4] Adra, S., Sun, T., MacNeil, S., Holcombe, M. and Smallwood, R. (2010) Development of a Three Dimensional Multiscale Computational Model of the Human Epidermis. Open Journal PLoS ONE, 5, e8511. https://doi.org/10.1371/journal.pone.0008511

[5] Nagayama, K., Nitta, J. and Miura, I. (2009) Numerical Analysis on Angiogenesis in Cancer Using a Particle Model. Theoretical and Applied Mechanics Japan, 58, 321-324.

[6] Nagayama, K., Matsuoka, S., Morisaki, N. and Taguchi, H. (2015) 3D Numerical Simulation of Hair Formation Process Using a Particle Model. Open Journal of Regenerative Medicine. http://file.scirp.org/Html/1-2390044_53849.htm

[7] Nagayama, K., Uehara, T., Amano, Y. and Tanahashi, M. (2015) 3D Numerical Simulation of Epidermis Turnover Process Using a Particle Model. Journal of Biosciences and Medicines.

[8] Nagayama, K., Kurihara, T., Amano, Y. and Tanahashi, M. (2016) Particle Simulation of Skin Basal Layer Formation. Journal of Biosciences and Medicines. http://file.scirp.org/Html/64656.html

[9] Kurihara, T. and Nagayama, K. (2017) Particle Simulation on Human Epidermal Aging-Effect of Basal Layer and Cell Division Rate. 7 th TSME-International Conference on Mechanical Engineering, BME001, Thailand.

[10] Shimizu, H.S. (2011) Textbook of Modern Dermatology. Nakayamashoten Co. 38, Japan.

[11] Hansen, S., Lehr, C.-M. and Schaefer, U.F. (2013) Improved Input Parameters for Diffusion Models of Skin Absorption. Advanced Drug Delivery Reviews, 65, 251-264. https://doi.org/10.1016/j.addr.2012.04.011

[12] Stockdale, M. (1978) Water Diffusion Coefficients versus Water Activity in Stratum Corneum: A Correlation and Its Implications. Journal of the Society of Cosmetic Chemists, 29, 625-639.

[13] Koyama, J. (1999) Mechanism of Adhesion and Detachment of Stratum Corneum and Its Role on Skin Care. Journal of Japanese Cosmetics Engineers, 33, 16-26.

[14] Takahashi, G. (2016) Efficacy Evaluation and Skin Measurement Useful for Research on Usefulness of Cosmetics and Foods, Chapter 3. Jihou Press, Japan. 